[3] R. Engelking and A. Lelek, Cartesian products and continuous images, Colloq. Math. 8 (1961), 27-29.

[4] K. Kuratowski, Topology, vols. I and II, Warsaw 1966 and 1968.

[5] R. Mańka, On uniquely arcwise connected curves, Colloq. Math. 51 (1987), 227-238.

[6] M. Štanko, Continua with the fixed point property, Dokl. Akad. Nauk SSSR 154 (1964) 1291-1293 (in Russian; translated in Soviet Math. 5 (1964), 303-305)

[7] A. L. Y andl, On a question concerning fixed points, Amer. Math. Monthly 75 (1968), 152-156. INSTYTUT MATEMATYCZNY PAN INSTITUTE OF MATHEMATICS Sniadeckich 8

Poland

\section{The nonexistence of expansive homeomorphisms of dendroids}

by

Hisao Ka to (Houston, Tex.)
Abstract. In this paper, we prove that no dendroid admits an expansive homeomorphism. Also, we show that no uniformly arcwise connected continuum admits an expansive homeomorphism.

0. Introduction. Let $X$ be a compact metric space with metric $d$. A homeomorphism $f$ of $X$ is expansive if there exists $c>0$ (called an expansive constant for $f$ ) such that $d\left(f^{n}(x), f^{n}(y)\right) \leqslant c$ for all integers $n$ implies $x=y$. This property is important in the topological theory of dynamical systems.

It is well known that the Cantor set, the 2-adic solenoid and the 2-torus admit expansive homeomorphisms ([11]).

Also, Bryant, Jacobson and Utz proved that there exists no expansive homeomorphism on an arc and a circle (see [1] and [6]). By using those results, Kawamura showed that if $X$ is a Peano continuum and $X$ contains a free arc, then $X$ admits no expansive homeomorphism ([7]). In [8], we showed that if $X$ is a Peano continuum which contains a 1-dimensional open ANR then $X$ does not admit an expansive homeomorphism. In particular, 1-dimensional compact ANRs admit no expansive homeomorphism. Also, Jacobson and Utz [6] asserted that the shift homeomorphism of the inverse limit of any continuous surjection of an arc is not an expansive homeomorphism (see [5] for a simple proof). The limit is a special type of arc-like continua and arc-like continua are tree-like. Naturally, the following problem arises: Is it true that no tree-like continuum admits an expansive homeomorphism?

The purpose of this paper is to prove that no dendroid (= arcwise connected tree-like continuum) admits an expansive homeomorphism, and no uniformly arcwise connected continuum admits an expansive homeomorphism.

1. Preliminaries. All spaces under consideration are assumed to be metric. A continuum is a compact connected nondegenerate space.

A continuum $X$ is said to be unicoherent provided that if $X=A \cup B$ and $A, B$ are subcontinua of $X$, the intersection $A \cap B$ is connected. A continuum $X$ is hereditarily

1980 Mathematics Subject Classification: Primary 54F50; Secondary 54H20, 58F15. 
unicoherent provided that each subcontinuum of $X$ is unicoherent. A continuum $X$ is tree-like provided that for any $\varepsilon>0$ there is a finite open cover $\mathscr{T}$ of $X$ such that the nerve $N(\mathscr{T})$ is a polyhedral tree and mesh $\mathscr{T}<\varepsilon$. A continuum $X$ is said to be a dendroid provided that $X$ is an arcwise connected hereditarily unicoherent space. It is well known that $X$ is a dendroid if and only if $X$ is an arcwise connected and tree-like continuum ([4]). Also, every nondegenerate subcontinuum of a dendroid is a dendroid. A continuum $X$ is said to be uniformly arcwise connected (cf. [3]) provided that it contains an arc and if for every number $\eta>0$ there is a natural number $n$ such that every arc $A$ in $X$ contains points $a_{0}, a_{1}, \ldots, a_{n}$ such that $A=\bigcup_{i=0}^{n-1}\left\langle a_{i} a_{i+1}\right\rangle$ and $\operatorname{diam}\left\langle a_{i} a_{i+1}\right\rangle\left\langle\eta\right.$ for each $i=0,1, \ldots, n-1$, where $\left\langle a_{i} a_{i+1}\right\rangle$ denotes the subarc from $a_{i}$ to $a_{i+1}$ in $A$. Note that every 1-dimensional compact connected ANR is uniformly arcwise connected and the 2-adic solenoid is not uniformly arcwise connected.

(1.1) EXAMPLE. In the Euclidean plane $E^{2}$, let $[a, b]$ denote the segment from $a$ to $b$, where $a, b \in E^{2}$. Consider the following points in $E^{2}$ :

$$
\begin{gathered}
p=(0,0), \quad a_{n}=\left(2,2 / 2^{n-1}\right) \quad(n=1,2, \ldots,), \\
a_{n}^{k}=\left(2,(n-k) / 2^{n} n+2 / 2^{n+1}\right), \quad b_{n}^{k}=\left(1,(n-k) / 2^{n+1} n+1 / 2^{n+1}\right) \\
(k=1,2, \ldots, n-1), \quad q=(2,0) .
\end{gathered}
$$

Let $X=\bigcup_{n=1}^{\infty}\left(\left[p, a_{n}\right] \cup\left[a_{n}, b_{n}^{1}\right] \cup\left[b_{n}^{1}, a_{n}^{1}\right] \cup \ldots \cup\left[b_{n}^{n-1}, a_{n}^{n-1}\right]\right) \cup[p, q]$ (see Figure 1).

Clearly, $X$ is a dendroid, but it is not uniformly arcwise connected.

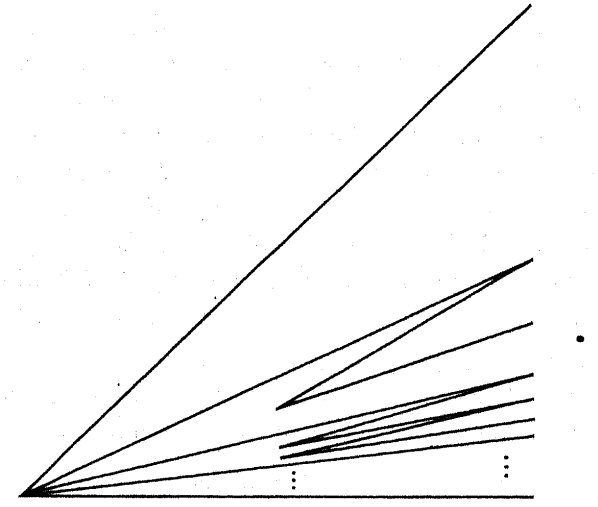

1.ig. 1

2. Expansive homeomorphisms and uniformly arcwise connected continua. In this section, we show that if $X$ is a uniformly arcwise connected continuum, then $X$ does not admit an expansive homeomorphism. We need the following.
(2.1) (Mañé [10]). Let $f: X \rightarrow X$ be an expansive homeomorphism of a compactum $X$ and let $c>0$ be an expansive constant for $f$ and $0<\varepsilon<c / 2$. Then there exists $\delta>0$ such that if $x, y \in X, d(x, y)<\delta$, and $\varepsilon \leqslant \sup \left\{d\left(f^{j}(x), f^{j}(y)\right) \mid 0 \leqslant j \leqslant n\right\} \leqslant 2 \varepsilon$ for some $n \geqslant 0$, then $d\left(f^{n}(x), f^{n}(y)\right) \geqslant \delta$.

(2.2) LeMMA. Let $f: X \rightarrow X$ be an expansive homeomorphism of a compactum $X$. Then there exists $\delta>0$ such that if $A$ is a nondegenerate subcontinuum of $X$, there exists a natural number $n_{0}\left(n_{0} \geqslant 0\right)$ such that one of the following conditions holds:

$$
\begin{array}{ll}
\operatorname{diam} f^{n}(A) \geqslant \delta & \text { for } n \geqslant n_{0} . \\
\operatorname{diam} f^{-n}(A) \geqslant \delta & \text { for }-n \leqslant-n_{0} .
\end{array}
$$

Proof. By (2.1), there is $\delta>0$ satisfying the conditions as in (2.1). Choose a subcontinuum $A^{\prime}$ of $A$ such that $\operatorname{diam} A^{\prime}<\delta$ and choose two points $a$ and $b$ in $A^{\prime}(a \neq b)$. Since $f$ is expansive, there is an integer $m \in Z$ such that $d\left(f^{m}(a), f^{m}(b)\right)>\varepsilon$. Suppose that $m \geqslant 0$. Set $n_{0}=m$. Let $n \geqslant n_{0}$. We must show that $\operatorname{diam} f^{n}(A) \geqslant \delta$. Choose $\eta>0$ such that $d(x, y)<\eta$ implies $d\left(f^{j}(x), f^{j}(y)\right)<\varepsilon$ for each $0 \leqslant j \leqslant n$. Since $A^{\prime}$ is connected, there is a finite sequence $a=a_{0}, a_{1}, \ldots, a_{p}=b$ of points in $A^{\prime}$ such that $d\left(a_{i}, a_{i+1}\right)<\eta$ for each $i$. For each $0 \leqslant r \leqslant p$, define $S_{r}=\sup \left\{d\left(f^{j}\left(a_{0}\right), f^{j}\left(a_{r}\right)\right) \mid 0 \leqslant j \leqslant n\right\}$. Note that $S_{0}=0$ and $S_{p}>\varepsilon$. Also, note that $\left|S_{r+1}-S_{r}\right| \leqslant \varepsilon$ for all $r$. Hence we can choose $r$ such that $S_{r-1} \leqslant \varepsilon$ and $S_{r}>\varepsilon$. Then $S_{r} \leqslant 2 \varepsilon$. By $(2.1), d\left(f^{n}\left(a_{0}\right), f^{n}\left(a_{r}\right)\right) \geqslant \delta$. Hence $\operatorname{diam} f^{n}(A) \geqslant \delta$. The case for $m<0$ is the same as above, because $f^{-1}$ is expansive as well.

(2.3) THEOREM. No uniformly arcwise connected continuum admits an expansive homeomorphism.

Proof. Let $X$ be a uniformly arcwise connected continuum. Suppose, on the contrary, that there exists an expansive homeomorphism $f$ on $X$. Choose an arc $A$ in $X$ Let $\delta>0$ be a positive number as in (2.2). Since $X$ is uniformly arcwise connected, there is a natural number $m$ such that (\#) if $B$ is an arc, there are points $b_{0}, b_{1}, \ldots, b_{m}$ of $B$ such that $B=\bigcup_{i=0}^{m-1}\left\langle b_{i}, b_{i+1}\right\rangle$ and $\operatorname{diam}\left\langle b_{i}, b_{i+1}\right\rangle\langle\delta$ for each $i$. Choose a sufficiently large natural number $p(p>2(m+1))$ and subarcs $A_{j}(j=1,2, \ldots, p)$ of $A$ such that

$$
A \supset \bigcup_{j=1}^{p} A_{j}, \quad A_{j} \cap A_{k}=\varnothing \quad(j \neq k) .
$$

Clearly, we may assume that $|J| \geqslant m+1$, where $J=\{j \in\{1,2, \ldots, p\} \mid$ there is a natural number $n_{j}$ such that $\operatorname{diam} f^{n}\left(A_{j}\right) \geqslant \delta$ for $\left.n \geqslant n_{j}\right\}$ and $|S|$ denotes the cardinal number of a set $S$. Put $n_{0}=\max \left\{n_{j} \mid j \in J\right\}$. Then the $\operatorname{arc} f^{n}(A)$ does not satisfy the condition (\#) if $n \geqslant n_{0}$. This is a contradiction.

(2.5) Corollary [8, (2.3) and (3.2)]. If $X$ is a 1-dimensional compact ANR, then $X$ admits no expansive homeomorphism.

3. The nonexistence of expansive homeomorphisms of dendroids. In this section, we prove the following main theorem in this paper. 
(3.1) THEOREM. No dendroid admits an expansive homeomorphism.

To prove (3.1), we need the following (cf. [9]).

Let $\delta>0$ be a positive number and let $n$ be a natural number $(n \geqslant 1)$. Let $C$ be an arc containing points $a, b$. Then $(C, a, b)$ is said to be $(\delta, n)$-folding provided that there exist points $a=x_{0}<x_{1}<\ldots<x_{n}=b$ in $C$ such that $\operatorname{diam}\left\langle x_{i}, x_{i+1}\right\rangle \geqslant \delta$ for each $i=0,1, \ldots, n-1$. Let $A, B, C$ be subsets of $X$. A triple $(A, B, C)$ is contained in a triple $\left(A^{\prime}, B^{\prime}, C^{\prime}\right)$ provided that $A \subset A^{\prime}, B \subset B^{\prime}$ and $C \subset C^{\prime}$. Moreover, if $A^{\prime}, B^{\prime}$ and $C^{\prime}$ are neighborhoods of $A, B$, and $C$, respectively, then we call $\left(A^{\prime}, B^{\prime}, C^{\prime}\right)$ a neighborhood of $(A, B, C)$.

Consider the following set:

$$
D(X)=\{(A, x, y) \in C(X) \times X \times X \mid x, y \in A\},
$$

where $C(X)$ denotes the hyperspace of subcontinua of $X$ with the Hausdorff metric

For any subset $M \subset D(X)$ and any $\delta>0$, define

$M f=\{(A, x, y) \in D(X) \mid$ for each neighborhood $(G, U, V)$ of $(A, x, y)$ and for any natural number $n$, there exists $\left(A^{\prime}, x^{\prime}, y^{\prime}\right) \in M$ such that $(G, U, V)$ is a neighborhood of $\left(A^{\prime}, x^{\prime}, y^{\prime}\right)$ and any arc from $x^{\prime}$ to $y^{\prime}$ in $A^{\prime}$ is $(\delta, n)$-folding?.

In this case, we say that $\left(A^{\prime}, x^{\prime}, y^{\prime}\right)$ is $(\delta, n)$-folding. For simplicity, set $M \delta=M^{f}$.

(3.2) Proposition. $M^{f}$ is closed in $D(X)$

(3.3) Proposition. $\left(M^{f}\right)^{f} \subset M^{f}$.

For a subset $M$ of $D(X)$ and for ordinal numbers, define

$$
M_{1}=M^{f}, \quad M_{\alpha+1}=\left(M_{\alpha}\right)^{f}, \quad M_{\lambda}=\bigcap_{\alpha<\lambda} M_{\alpha}, \quad \text { where } \lambda \text { is a limit ordinal. }
$$

(3.4) THEOREM. If $X$ is a dendroid, then $M_{\alpha}=\varnothing$ for some countable ordinal $\alpha$.

Proof. Note that $D(X)$ is separable. Since $M_{\alpha}$ is closed in $D(X)$ and $M_{\alpha} \supset M_{\beta}$ for $\beta \geqslant \alpha$, there is a countable ordinal $\alpha$ such that $M_{\alpha}=M_{\beta}$ for all $\beta \geqslant \alpha$. In particular, $\left(M_{\alpha}\right)^{f}=M_{\alpha}$. We shall show that $M_{\alpha}=\varnothing$. Suppose, on the contrary, that $M_{\alpha} \neq \varnothing$. Choose $\left(A_{1}, x_{1}, y_{1}\right) \in M_{\alpha}$. Since $A_{1}$ is a tree-like continuum, there is a finite open cover $\mathscr{I}_{1}$ of $A_{1}$ such that

a(1) $G_{1}=\bigcup \mathscr{I}_{1}\left(=\bigcup\left\{W \in \mathscr{I}_{1}\right\}\right)$ is a neighborhood of $A_{1}$,

b(1) $\operatorname{mesh} \mathscr{I}_{1}<1 / 2$,

c(1) the nerve $N\left(\mathscr{I}_{1}\right)$ is a tree, and

$\mathrm{d}(1) x_{1} \in U_{1} \in \mathscr{I}_{1}$ and $y \in V_{1} \in \mathscr{I}_{1}$.

Since $\left(A_{1}, x_{1}, y_{1}\right) \in\left(M_{\alpha}\right)^{f}$, we can choose $\left(A_{2}, x_{2}, y_{2}\right) \in M_{\alpha}$ such that $\left(G_{1}, U_{1}, V_{1}\right)$ is a neighborhood of $\left(A_{2}, x_{2}, y_{2}\right)$ and $\left(A_{2}, x_{2}, y_{2}\right)$ is $(\delta, 1)$-folding. Take a finite open cover $\mathscr{I}_{2}$ of $A_{2}$ such that

a(2) $G_{2}=\bigcup \mathscr{I}_{2}$ is a neighborhood of $A_{2}$ and $\mathrm{Cl} G_{2} \subset G_{1}$

b(2) $\operatorname{mesh} \mathscr{I}_{2}<1 / 2^{2}$,

c(2) $N\left(\mathscr{I}_{2}\right)$ is a tree,

$\mathrm{d}(2) x_{2} \in U_{2} \in \mathscr{I}_{2}, y_{2} \in V_{2} \in \mathscr{I}_{2}$ and $\mathrm{Cl} U_{2} \subset U_{1}, \mathrm{Cl} V_{2} \subset V_{1}$ and

$\mathrm{e}(2)\left(N\left(\mathscr{I}_{2}\right), U_{2}, V_{2}\right)$ is $(\delta, 1)$-folding, where, in general, $((N), \mathscr{Y}, U, V)$ is said to be $(\delta, n)$-folding for a cover $\mathscr{I}$ with $U, V \in \mathscr{I}$ provided that for any chain $\mathscr{A}$ from $U$ to $V$, there are $U=W_{0}<W_{1}<\ldots<W_{n}=V$ in $\mathscr{A}$ such that $\operatorname{diam}\left(\bigcup \mathscr{\mathscr { A } _ { i }}\right) \geqslant \delta(i=0,1, \ldots, n-1)$, where $\mathscr{A}_{1}$ is the subchain from $W_{i}$ to $W_{i+1}$ in.$\%$ (see Figure 2).

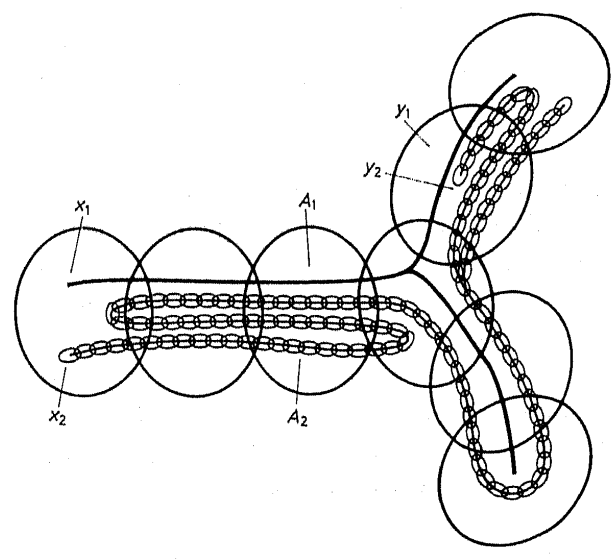

Fig. 2

By induction, we obtain $\left(A_{i}, x_{i}, y_{i}\right) \in M_{\alpha}$ and a finite open cover $\mathscr{I}_{i}$ of $A_{i}$ such that $\left(G_{i-1}, U_{i-1}, V_{i-1}\right)$ is a neighborhood of $\left(A_{i}, x_{i}, y_{i}\right),\left(A_{i}, x_{i}, y_{i}\right)$ is $(\delta, i)$-folding and $\mathscr{I}_{i}$ satisfies the following;

a(i) $G_{i}=\bigcup \mathscr{I}_{i}$ is a neighborhood of $A_{i}$ and $\mathrm{Cl} G_{i} \subset G_{i-1}$,

b(i) $\operatorname{mesh} \mathscr{I}_{i}<1 / 2^{i}$,

c(i) $N\left(\mathscr{I}_{i}\right)$ is a tree,

$\mathrm{d}(\mathrm{i}) x_{i} \in U_{i} \in \mathscr{I}_{i}, y_{i} \in V_{i} \in \mathscr{I}_{i}$ and $\mathrm{Cl} U_{i} \subset U_{i-1}, \mathrm{Cl} V_{i} \subset V_{i-1}$,

e(i) $\left(N\left(\mathscr{I}_{i}\right), U_{i}, V_{i}\right)$ is $(\delta, i)$-folding.

Put $(A, x, y)=\left(\bigcap_{i=1}^{\infty} \mathrm{Cl} G_{i}, \bigcap_{i=1}^{\infty} \mathrm{Cl} U_{i}, \bigcap_{i=1}^{\infty} \mathrm{Cl} V_{i}\right)$. Then $A$ is a continuum. Since $X$ is a dendroid, $A$ is a dendroid. Then there is an $\operatorname{arc} C$ from $x$ to $y$ in $A$. By the construction, $(C, x, y)$ is $(\delta, i)$-folding for all $i$. This is impossible.

Pro of of (3.1). Let $X$ be a dendroid. Suppose, on the contrary, that $X$ admits an expansive homeomorphism $f: X \rightarrow X$. By (3.4), there is a countable ordinal $\alpha$ such that $M_{\alpha}=\varnothing$, where $M=D(X)$. Let $c>0$ be an expansive constant and $0<\varepsilon<c / 2$. Choose $\delta>0$ satisfying the condition as in (2.2). Let $0<\eta<\delta / 2$. Take an $\operatorname{arc} A=B$ from $x$ to $y=y^{\prime}$. We may assume that $d(x, y) \geqslant \eta$. Let $m_{1}<m_{2}<\ldots$, be a sequence of natural numbers. By the proof of $(2.3)$, there exists a sequence $n(1), n(2), \ldots$, of integers $Z$ with $|n(1)|<|n(2)|<\ldots$, satisfying orle of the following conditions:

$\Delta(1)$ if $n(i) \geqslant 0$, for all $n \geqslant n(i),\left(f^{n}(B), f^{n}(x), f^{n}\left(y^{\prime}\right)\right)$ is $\left(\delta, m_{i}+1\right)$-folding.

$\nabla(1)$ if $n(i)<0$, for all $n \leqslant n(i),\left(f^{n}(B), f^{n}(x), f^{n}\left(y^{\prime}\right)\right)$ is $\left(\delta, m_{i}+1\right)$-folding. 
For each $i$, take a point $a_{i}$ of $\left\langle f^{2 n(i)}(x), f^{2 n(i)}\left(y^{\prime}\right)\right\rangle$ such that $\operatorname{diam}\left\langle f^{2 n(i)}(x), a_{i}\right\rangle \geqslant \delta$, and $\left(\left\langle a_{i}, f^{2 n(i)}\left(y^{\prime}\right)\right\rangle, a_{i}, f^{2 n(i)}(y)\right)$ is $\left(\delta, m_{i}\right)$-folding. Since $C(X)$ is a compact metric space, we may assume that $\lim f^{2 n(i)}(A)=A_{1}, \lim f^{2 n(i)}(y)=y_{1}$ and $\lim \left\langle f^{2 n(i)}(x), a_{i}\right\rangle=B_{1}^{\prime}$. Note that $B_{1}^{\prime} \subset A_{1}$. Since diam $B_{1}^{\prime} \geqslant \delta>2 \eta$, there is a point $x_{1}$ of $B_{1}^{\prime}$ and an arc $B_{1}\left(x_{1} \in B_{1}\right)$ such that $B_{1} \subset B_{1}^{\prime}$ and $\left\langle x_{1}, y_{1}\right\rangle \cap B_{1}=B_{1}$. Let $B_{1}=\left\langle x_{1}, y_{1}^{\prime}\right\rangle$. We may assume that $d\left(x_{1}, y_{1}^{\prime}\right) \geqslant \eta$, i.e., $\operatorname{diam} B_{1} \geqslant \eta$. For a countable ordinal $\lambda$, we assume that we have obtained $\left(A_{\alpha}, x_{\alpha}, y_{\alpha}\right) \in D(X), B_{\alpha}^{\prime}$ and $B_{\alpha}$ for all $\alpha<\lambda$ as before. Let $y_{\alpha}^{\prime}$ be the point $\left\langle x_{\alpha}, y_{\alpha}^{\prime}\right\rangle=B_{\alpha}$.

Consider two cases:

(I) $\lambda=\alpha+1$. As before, we have a sequence $n(1), n(2), \ldots$, of integers $Z$ such that $|n(1)|<|n(2)|<, \ldots$, and one of the following conditions holds:

$\Delta(\lambda)$ If $n(i) \geqslant 0$, then $\left(f^{n}\left(B_{\alpha}\right), f^{n}\left(x_{\alpha}\right), f^{n}\left(y_{\alpha}^{\prime}\right)\right)$ is $\left(\delta, m_{i}+1\right)$-folding for all $n \geqslant n(i)$.

$\nabla(\lambda)$ If $n(i)<0$, then $\left(f^{n}\left(B_{\alpha}\right), f^{n}\left(x_{\alpha}\right), f^{n}\left(y_{\alpha}^{\prime}\right)\right)$ is $\left(\delta, m_{t}+1\right)$-folding for all $n \leqslant n(i)$.

For each $i$, choose a point $a_{i}$ of $f^{2 n(i)}\left(B_{\alpha}\right)$ such that $\operatorname{diam}\left\langle f^{2 n(i)}\left(x_{\alpha}\right), a_{i}\right\rangle \geqslant \delta$ and $\left(\left\langle a_{i}, f^{2 n(i)}\left(y_{\alpha}^{\prime}\right)\right\rangle, a_{i}, f^{2 n(i)}\left(y_{\alpha}^{\prime}\right)\right)$ is $\left(\delta, m_{i}\right)$-folding, hence $\left(\left\langle a_{i}, f^{2 n(i)}\left(y_{\alpha}\right)\right\rangle, a_{i}, f^{2 n(i)}\left(y_{\alpha}\right)\right)$ is $\left(\delta, m_{i}\right)$-folding. We may assume that the sequences $\left\{f^{2 n(i)}\left(A_{\alpha}\right)\right\}_{i},\left\{\left\langle f^{2 n(i)}\left(x_{\alpha}\right), a_{i}\right\rangle\right\}_{i}$ and $\left\{f^{2 n(i)}\left(y_{\alpha}\right)\right\}_{i}$ are convergent in $C(X)$. Set $A_{\alpha+1}=\lim f^{2 n(i)}\left(A_{\alpha}\right), y_{\alpha+1}=\lim f^{2 n(i)}\left(y_{\alpha}\right)$ and $B_{\alpha+1}^{\prime}=\lim \left\langle f^{2 n(i)}\left(x_{\alpha}\right), a_{i}\right\rangle$. Then $\operatorname{diam} B_{\alpha+1}^{\prime} \geqslant \delta$. Choose a point $x_{\alpha+1}$ of $B_{\alpha+1}^{\prime}$ and an arc $B_{\alpha+1}$ in $B_{\alpha+1}^{\prime}$ such that $x_{\alpha+1} \in B_{\alpha+1},\left\langle x_{\alpha+1}, y_{\alpha+1}\right\rangle \cap B_{\alpha+1}=B_{\alpha+1}$ and $d\left(x_{\alpha+1}, y_{\alpha+1}^{\prime}\right) \geqslant \eta$ i.e., $\operatorname{diam} B_{\alpha+1} \geqslant \eta$, where $B_{\alpha+1}=\left\langle x_{\alpha+1}, y_{\alpha+1}^{\prime}\right\rangle$.

(II) $\lambda$ is a limit ordinal. In this case, take a sequence $\alpha_{1}<\alpha_{2}<\ldots$ of countable ordinal numbers such that $\lim \alpha_{i}=\lambda$. Then we may assume that the sequences $\left\{A_{\alpha_{1}}\right\}$, $\left\{y_{\alpha_{i}}\right\}$ and $\left\{B_{\alpha_{i}}^{\prime}\right\}$ are convergent. Set $A_{\lambda}=\lim A_{\alpha_{i}}, y_{\lambda}=\lim y_{\alpha_{i}}$ and $B_{\lambda}^{\prime}=\lim B_{\alpha_{i}}^{\prime}$. Also, choose a point $x_{\lambda}$ of $B_{\lambda}^{\prime}$ and an arc $B_{\lambda}$ such thät $x_{\lambda} \in B_{\lambda},\left\langle x_{\lambda}, y_{\lambda}\right\rangle \cap B_{\lambda}=B_{\lambda}$ and $\operatorname{diam} B_{\lambda} \geqslant \eta$. Let $B_{\lambda}=\left\langle x_{\lambda}, y_{\lambda}^{\prime}\right\rangle$. Hence we obtain $\left(A_{\alpha}, x_{\alpha}, y_{\alpha}\right) \in D(X), B_{\alpha}^{\prime}$ and $B_{\alpha}$ for all countable ordinal numbers $\alpha$.

Next, we shall show that for each countable ordinal number $\alpha$,

$$
\left(f^{n}\left(A_{\alpha}\right), f^{n}(b), f^{n}\left(y_{\alpha}\right)\right) \in M_{\alpha}
$$

where $M=D(X), M^{\mathcal{f}}=M_{\delta}^{f}, b \in B_{\alpha}^{\prime}$ and $n$ is any integer

We shall prove this by transfinite induction. Let $(G, U, V)$ be a neighborhood of $\left(f^{n}\left(A_{1}\right), f^{n}(b), f^{n}\left(y_{1}\right)\right)$. Since $\left(f^{-n}(G), f^{-n}(U), f^{-n}(V)\right)$ is a neighborhood of $\left(A_{1}, b, y_{1}\right)$, where $b \in B_{1}^{\prime}$, we can choose $n(i)$ such that if $n(i) \geqslant 0,2 n(i)+n \geqslant n(i)$, or if $n(i)<0$, $2 n(i)+n \leqslant n(i)$, and $\left(f^{2 n(i)}(A), b_{i}, f^{2 n(i)}(y)\right)$ is contained in $\left(f^{-n}(G), f^{-n}(U), f^{-n}(V)\right)$, where $b_{i} \in\left\langle f^{2 n(i)}(x), a_{i}\right\rangle$ such that $\lim b_{i}=b$. By $\Delta(1)$ or $\nabla(1),\left(f^{2 n(i)+n}(A), f^{n}\left(b_{i}\right)\right.$, $\left.f^{2 n(i)+n}(y)\right)$ is $\left(\delta, m_{i}\right)$-folding. Hence $\left(f^{n}\left(A_{1}\right), f^{n}(b), f^{n}\left(y_{1}\right)\right) \in M_{1}$

Assume that for each integer $n$,

$$
\left(f^{n}\left(A_{\alpha}\right), f^{n}(b), f^{n}\left(y_{\alpha}\right)\right) \in M_{\alpha} \quad \text { for all } \alpha<\lambda \text {, where } b \in B_{\alpha}^{\prime} .
$$

Consider two cases:

(I) $\lambda=\alpha+1$. Let $(G, U, V)$ be any neighborhood of $\left(f^{n}\left(A_{2}\right), f^{n}(b), f^{n}\left(y_{2}\right)\right)$, where $b \in B_{\lambda}^{\prime}$. By the construction, we can choose a large number $k>0$ such that $\left(f^{2 n(k)}\left(A_{\alpha}\right), b_{k}, f^{2 n(k)}\left(y_{\alpha}\right)\right)$, where $b_{k} \in\left\langle f^{2 n(k)}\left(x_{\alpha}\right), a_{k}\right\rangle \subset f^{2 n(k)}\left(B_{\alpha}\right)$ and $\lim b_{k}=b$, is $\left(\delta, m_{k}\right)$-folding and is contained in $\left(f^{-n}(G), f^{-n}(U), f^{-n}(V)\right)$. By the construction, $\left(f^{2 n(k)+n}\left(A_{\alpha}\right), f^{n}\left(b_{k}\right), f^{2 n(k)+n}\left(y_{\alpha}\right)\right)$ is $\left(\delta, m_{k}\right)$-folding and is contained in $M_{\alpha}$ by the inductive hypothesis. Hence $\left(f^{n}\left(A_{\lambda}\right), f^{n}(b), f^{n}\left(y_{\lambda}\right)\right) \in M_{\lambda}$.

(II) $\lambda$ is a limit ordinal. By an argument similar to the above one, we can prove that

$$
\left(f^{n}\left(A_{\lambda}\right), f^{n}(b), f^{n}\left(y_{\lambda}\right)\right) \in M_{\alpha} \quad \text { for } \alpha<\lambda, b \in B_{\lambda}^{\prime} .
$$

Hence $\left(f^{n}\left(A_{\lambda}\right), f^{n}(b), f^{n}\left(y_{\lambda}\right)\right) \in \bigcap_{\alpha<\lambda} M_{\alpha}=M_{\lambda}$.

In particular, $M_{\alpha} \neq \varnothing$ for all countable ordinal numbers $\alpha$. This is in contradiction to (3.4). This completes the proof.

The following problem remains open.

Problem. If a continuum $X$ is tree-like (moreover arc-like), is it true that $X$ does not admit an expansive homeomorphism?

\section{References}

[1] B. F. Bryant, Unstable self-homeomorphisms of a compact spaces, Vanderbilt University Thesis, 1954.

[2] J. J. Charatonik, On ramification points in the classical sense, Fund. Math. 51 (1962), 229-252.

[3] - Two invariants under continuity and the incomparability of fans, Fund. Math. 53 (1964), 187-204.

[4] H. Cook, Tree-likeness of dendroids and $\lambda$-dendroids, Fund. Math. 68 (1970), 19-22.

[5] M. Dateyama, Some class of one-dimensional dynamical systems without expansiveness, preprint.

[6] J. F J 2-cell, Pacific J. Math. 10 (1960), 1319-1321.

[7] K. Kawamura, $A$ direct proof that each Peano continuum with a free arc admits no expansive homeomorphisms, Tsukuba J. Math., 12 (1988), 521-524.

[8] $\mathrm{H}$. Kato, The nonexistence of expansive homeomorphisms of 1-dimensional compact ANRs, Proc. Amer. Math. Soc., to appear.

[9] J. K rasinkiew and P. Minc, Nonexistence of universal continua for certain classes of curves, Bull. Acad. Polon. Math. 24 (1976), 733-741.

[10] R. M a ñé, Expansive homeomorphisms and topological dimension, Trans. Amer. Math. Soc. 252 (1979), 313-319.

[11] R. F. Williams, A note on unstable homeomorphisms, Proc. Amer. Math. Soc. 6 (1955) 308-309.

DEPARTMENT OF MATHEMATICS

UNIVERSITY OF HOUSTON

Houston, Texas 77204-3476

U.S.A.

Current address:

FACULTY OF INTEGRATED ARTS AND SCIENCES

ENOSHMA UNIVERSITY

Hiroshima 730, Japan

Received 11 January 1989 